\title{
ABUSO SEXUAL INFANTO-JUVENIL EM UMA ANÁLISE SOBRE TÉCNICAS EM TERAPIAS COGNITIVO-COMPORTAMENTAIS EM GRUPO (TCCG)
}

\author{
Késia Alves Silva ${ }^{1}$ \\ Antonio Leonardo Figueiredo $\mathrm{Calou}^{2}$ \\ Rivalina Maria Macêdo Fernandes ${ }^{3}$
}

Resumo: Este trabalho teve por objetivo promover a reflexão, o estudo e a pesquisa acerca da temática do abuso sexual na infância e na adolescência, tendo como luz a terapia cognitivacomportamental. Diante do crescente número de casos, visualizamos a necessidade de se redobrar a assistência psicológica. Sabendo do importante impacto gerado por este fenômeno a inúmeras crianças e adolescentes, foi pontuada sobre o importante papel do psicólogo no acolhimento a essas vítimas, bem como um eficaz acompanhamento em longo prazo, capaz de trazer uma nova ressignificação de vida, sofrimento e culpa. Em virtude disto, buscou-se aqui apresentar as técnicas mais utilizadas no acolhimento de crianças e adolescentes vítimas de abuso sexual, envolvidas no processo terapêutico grupal, realizado por profissionais psicólogos cognitivos e comportamentais.

\section{Palavras-Chave: Crianças e} Adolescentes. Abuso Sexual. Terapia Cognitivo-Comportamental.

\begin{abstract}
This study aimed to promote reflection, study and research on the theme of sexual abuse in childhood and adolescence, with cognitive behavioral therapy as its light. Given the growing number of cases, we see the need to redouble psychological assistance. Knowing the important impact generated by this phenomenon to countless
\end{abstract}

\footnotetext{
${ }^{1}$ Bacharel em Psicologia e especialista em Terapia Cognitivo-Comportamental pelo Centro Universitário INTA (UNINTA).

${ }^{2}$ Doutorando em Psicologia pela Universidade Federal do Rio Grande do Norte (UFRN). Cientista Social com mestrado na área. Professor do curso de especialização em Terapia Cognitivo-Comportamental do Centro Universitário INTA (UNINTA).

${ }^{3}$ Mestre em Gestão de Organizações Aprendentes pela Universidade Federal da Paraíba (UFPB). Psicóloga e professora do Centro Universitário INTA (UNINTA).
} 
children and adolescents, was stressed about the important role of the psychologist in welcoming these victims, as well as an effective long-term follow-up, capable of bringing a new resignification of life, suffering and guilt. Because of this, we sought to present the most used techniques in the reception of children and adolescents victims of sexual abuse, involved in the group therapeutic process, performed by professional cognitive and behavioral psychologists.

Keywords: Children and Adolescents. Sexual abuse. Cognitive behavioral therapy.

\section{Introdução}

A violência contra crianças e adolescentes constitui atualmente um problema de saúde pública que ocorre em diversas culturas e a todo momento. (Pelisoli e Picolloto, 2010). Ela não somente fere os direitos humanos de suas vítimas, mas também deixa inúmeras consequências deletérias para o desenvolvimento psicológico e físico da população infanto-juvenil. Dentre as formas de violência mais comuns podemos citar a negligência e abuso físico, psicológico e sexual que na maioria das vezes ocorrem no próprio ambiente familiar da criança (Habigzang e Koller, 2011).

De acordo com o Plano Nacional de Enfrentamento da Violência Sexual de Crianças e Adolescentes (Brasil, 2015), deve-se "trabalhar a violência sexual como um macroconceito que envolve duas expressões: abuso sexual e exploração sexual". Entendendo o abuso sexual, como tema central deste trabalho, partirmos da definição de Morechi (2018, p.17) que introduz como "toda ação que obriga uma pessoa a manter contato sexual, físico ou verbal, com uso da força, coerção, chantagem, manipulação, ameaça ou qualquer outro meio que anule ou limite a vontade pessoal". Já a exploração sexual, por sua vez, "é o uso sexual de criança ou adolescente para obter lucro, troca ou vantagem. Já a exploração sexual se expressa de quatro formas: prostituição, pornografia, tráfico e turismo sexual", segundo a cartilha do Ministério Público do Distrito Federal e Territórios (Brasil, 2015).

Conforme dados da Fundação ABRINQ - Associação Brasileira dos Fabricantes de Brinquedos, em 2017, o disque 100 recebeu mais de 144 mil 
denúncias de violação de direitos contra crianças e adolescentes em todo país. Dentre elas, 20,6\% foram casos de violência sexual contra crianças e adolescentes (Moreschi, 2018). Uma característica relevante e alarmante é a grande diferença no número de vítimas por sexo, sendo a criança do sexo feminino, a maior vítima de crimes sexuais. Ainda segundo esta Fundação, cerca de dois terços do total dos casos de abuso sexual registrados no Brasil, $70 \%$ é praticada contra o gênero feminino (Brasil, 2017).

Nesse contexto, visualizamos a necessidade de se redobrar a assistência psicológica, quanto às repercussões em longo prazo para o desenvolvimento físico e emocional de crianças e adolescentes expostas a este tipo de violência. De acordo Lira et al. (2017), a exposição ao abuso sexual na infância está associada a prejuízos futuros representado como fator de risco para o desencadeamento de depressão, ideias suicidas, ansiedade e transtorno do estresse pós-traumático.

Sabendo do imenso impacto gerado por este fenômeno a inúmeras crianças e adolescentes, reconhecemos o importante papel do psicólogo no acolhimento a essas vítimas, bem como um eficaz acompanhamento em longo prazo, capaz de trazer uma nova ressignificação de vida, amenizando as sequelas e seus efeitos. Assim, diante disso, pensa-se na seguinte questão: Em virtude desta demanda, quais as técnicas mais utilizadas e que são consideradas eficazes, no acolhimento de crianças e adolescentes vítimas de abuso sexual, envolvidas no processo terapêutico, realizado por profissionais psicólogos cognitivos comportamentais?

Diante de tais inquietações, propomos fazer aqui um estudo exploratório que segundo Gerhardt e Silveira (2009), tem como objetivo proporcionar maior familiaridade com a problemática em questão, objetivando torná-la mais explícita ou auxiliar na construção de hipóteses. Assim, este estudo baseia-se em materiais que foram publicados com propósito de analisar posições diversas em relação ao assunto (Gil, 2010).

Neste segmento, o estudo envolveu uma revisão bibliográfica de natureza qualitativa que teve o intentio de produzir todo o percurso da sua elaboração por meio de uma busca sistemática por informações, com o propósito de compreender para interpretar e explicar a realidade 
investigada. Para tal, Gil (2010) ratifica que se trata de um tipo de pesquisa construída através do levantamento de publicações preexistentes, utilizando principalmente artigos científicos e livros. Assim, buscou-se apresentar conceitos, prevalências, dados gerais e específicos acerca da temática, a fim de obter uma percepção mais ampla e embasada diante das demandas que transcorre o abuso sexual infanto-juvenil e o embasamento teórico e técnico à luz da terapia cognitivo-comportamental.

Os dados foram coletados entre os meses de Fevereiro, Março e Abril de 2019, nos bancos de dados do SCIELO, GOOGLE ACADÊMICO e PUBMED, acerca do tema. $\mathrm{O}$ material foi rastreado utilizando os descritores: "abuso sexual", "criança", "adolescente", "terapia cognitivo-comportamental" e “psicoterapia de grupo".

Como técnica de coleta de dados utilizou-se o fichamento, que segundo Marconi e Lakatos (2010), possibilita uma seleção constante da documentação e de seu ordenamento. Permitindo assim, selecionar dados relevantes para a pesquisa, empregando como critérios de inclusão e exclusão, informações relacionadas à expressão supracitada com enfoque na infância e ra adolescência das vítimas.

Seguindo os critérios utilizados foram encontrados inúmeros artigos referenciando o tema abuso sexual, porém com material escasso envolvendo as técnicas cognitivo-comportamentais. Após ter lido os resumos dos artigos foram escolhidos os que se assemelhavam com o tema, trazendo informações e contribuições para a pesquisa.

Analisamos esse material e desenvolvemos um texto que se dividiu em três momentos. O primeiro constituise de uma leitura sobre o tema, onde analisamos todo o contexto relacionado ao abuso sexual infanto-juvenil, desde sua origem no cenário nacional e internacional. O segundo capítulo tratase de uma perspectiva psicológica, interconectando-a com a terapia cognitiva comportamental como modelo efetivo no tratamento do público já mencionado acima. E o terceiro e último capítulo, vem contribuir com uma análise das técnicas mais utilizadas e que são consideradas eficazes, no tratamento psicológico diante do acolhimento dessas crianças e adolescentes, na perspectiva cognitivo-comportamental. 
O abuso sexual na infância e na adolescência

O abuso sexual enquanto um fenômeno multifacetado e complexo que afeta crianças e adolescentes, compreendido desde as tentativas de atentado ao pudor, até chegar ao ato do estupro, é considerado pela Organização Mundial da Saúde (OMS, 2002) como um problema de saúde pública mundial, configurando-se como uma das principais formas de violação de direitos, com desdobramentos e efeitos que afetam de forma impactante na saúde física e psíquica das vítimas.

De acordo com a OMS (2002), abuso sexual infantil é quando há o envolvimento de uma criança em atividade sexual que a mesma não compreende de forma completa, sendo assim incapacitada de consentir, ou para a qual, em função de seu desenvolvimento, a criança não está preparada e não pode permitir, violando assim seus direitos. Essa prática é evidenciada por estas atividades entre uma criança e um adulto ou outra criança, que, em razão da idade ou do nível de desenvolvimento, está em uma relação de responsabilidade, confiança ou poder (OMS, 2002).
Compreende-se como uma violência complexa de difícil suspeita e confirmação, estando os casos presentes na realidade global, sendo praticados, na grande maioria, por pessoas ligadas diretamente às vítimas. Embora nem sempre sejam acompanhados de violência física aparente, apresenta-se de diversas maneiras e níveis de gravidade, dificultando assim as denúncias por parte das vítimas e a confirmação pelos órgãos competentes.

De acordo com relatório do Fundo das Nações Unidas para a Infância - UNICEF, em 2005 infere-se que 150 milhões de meninas e 73 milhões de meninos com menos de 18 anos já sofreram relações sexuais violentadas ou outra forma de violência sexual ou física. Segundo o relatório "Situação da Infância Brasileira", ainda da UNICEF (2005), cerca de $20 \%$ das mulheres e $10 \%$ dos homens no mundo, já sofreram violência sexual na infância. Os dados revelam que $64 \%$ dos casos de abuso sexual contra crianças e adolescentes de até seis anos de idade sejam cometidos por pais ou familiares próximos, indicando que as crianças do sexo feminino estão mais vulneráveis do que as do sexo masculino (Saffioti, 1997). Os principais abusadores são pessoas do 
sexo masculino, sobretudo pessoas próximas, como pais, padrinhos, dentre outros (Capitão e Romaro, 2008). Desta forma, temos o ambiente doméstico como um espaço de violência sexual recorrente. Tal vínculo familiar contribui para que os dados estejam inebriados de obscuridade e o abuso dificilmente denunciado (Ribeiro, Ferriani e Reis, 2004). Entretanto as estatísticas ainda são inconclusivas, uma vez que a real prevalência é desconhecida, visto que, na maioria dos casos, as crianças e adolescentes não conseguem romper o silêncio, chegando a revelar o ocorrido apenas na idade adulta, o que impede um panorama mais fidedigno (Braun, 2002). Trata-se de uma violência perpassada pelo silêncio.

Fazendo um recorte no cenário nacional, existem poucos dados no Brasil sobre esta temática, uma lacuna que dificulta a real proporção de crianças e adolescentes afetados pelo abuso, como também os fatores que são relativos à sua ocorrência. Outro recorte que reforça esse precedente é o baixo número de notificações sobre a violência, com estimativa de que apenas $10 \%$ dos casos são de fato notificados (Barros, 2004).

Por ser um fenômeno global e de abrangência universal, varia de acordo com os diversos padrões culturais e especificidades sociais dos contextos de ocorrência, entretanto independente dessas questões refere-se a uma violação dos direitos essenciais das crianças e adolescentes, em caráter potencialmente danoso, com impactos significativos para as vítimas em todo o seu processo de desenvolvimento (Araújo, 2002).

Dessa forma, fez-se necessário o planejamento, organização e execução de políticas públicas destinadas ao cuidado e proteção, bem como a prevenção da violência sexual contra crianças e adolescentes, uma vez que demonstrou ao longo dos anos, ser um forte agravo a ser enfrentado.

Assim, o marco inicial de enfrentamento a essa violência em caráter internacional deu-se em 1996, com o I Congresso Mundial Contra a Exploração Sexual Comercial de Crianças e Adolescentes, realizado na Suécia, passando também a reconhecer a violação para com crianças e/ou adolescentes. Nacionalmente, o Ministério da Saúde (Brasil, 1999), normatizou a prevenção e tratamento dos agravos resultantes da violência sexual contra menores, onde em 2001, o Ministério da Justiça buscou traçar um Plano Nacional de Enfrentamento da 
Violência Sexual Infanto-Juvenil, como iniciativa do Governo Federal para a articulação da rede de proteção e no intuito da defesa dos direitos das crianças e adolescentes (Brasil, 2003).

As ações protetivas são embasadas nos princípios defendidos pelo Estatuto da Criança e do Adolescente no sentido de construir práticas interventivas para combater a essa violência, sejam elas através de atendimentos especializados às vítimas, ações preventivas, ou a busca pelo fortalecimento do sistema de defesa e responsabilização (Abrapia $\left.{ }^{4}, \quad 2002\right)$. Vale destacar que a partir do ano 2000, foi instituído o dia 18 de maio como o Dia Nacional do Combate ao Abuso e Exploração Sexual Infanto-Juvenil pela Lei Federal 9.970. Enquanto medida protetiva, o cenário brasileiro conta com diversos dispositivos legalmente reconhecidos. O Estatuto da Criança e do Adolescente - ECA ou Lei 8.069/90 é o maior referencial para essas ações, estabelecendo toda uma política de

4 “A ABRAPIA trabalha na prevenção primária, secundária e terciária da violência contra a criança nas suas principais formas: violência doméstica, violência social e violência na escola (bullying)" (Abrapia, 2002).

5 O Programa Sentinela, criado em 2001, durante segunda gestão de Fernando atendimento a esse público por meio de ações governamentais e não governamentais (Zagaglia, 2004).

Destaca-se que pela lei supracitada, são regulamentados os conselhos-tutelares responsáveis pela formulação de políticas de proteção integral a infância, que articulam os órgãos públicos e iniciativas privadas para a concretização da política de proteção e desenvolvimento das crianças e adolescentes (Brasil, 2003). Reforça-se também a criação do Programa Sentinela em $2001^{5}$ que se propõe garantir o atendimento integral e especializado às crianças e aos adolescentes em situação de violência sexual (Pinheiro, 2006).

Dessa forma, uma rede de apoio estrutura-se e possibilita o suporte necessário para que se possa atravessar essa ocorrência de forma a minimizar ou até mesmo excluir os agravos, implicando a organização de um sistema protetivo, eficaz e assertivo. A "proteção social especial é voltada para indivíduos

Henrique Cardoso - FHC (1999-2003), sob a coordenação da então Secretaria de Estado da Assistência Social - SEAS - do Ministério da Previdência e Ação Social MPAS, é uma ação do governo federal, que tem como objetivo $o$ atendimento especializado às crianças e aos adolescentes, vítimas da violência sexual (Santos, 2010). 
ou famílias que tiveram seus direitos violados ou ameaçados por circunstâncias diversas, por exemplo, o abuso sexual, exigindo atuação interdisciplinar e especializada" $\left(\mathrm{CFP}^{6}\right.$, 2009). Entretanto, para além da proteção legal é imprescindível o suporte para com os aspectos psicológicos pertinentes ao abuso sexual, inserindo as demandas terapêuticas da criança e das famílias nessa rede de cuidados (Habigzang et al, 2006).

Diante dessa necessidade, buscamos na sequencia estreitar os laços entre a psicologia e a terapia cognitivocomportamental, buscando os efeitos eficazes nos modelos de intervenção terapêutica para crianças e adolescentes.

\section{A psicologia e a terapia cognitivo-} comportamental como efetivo modelo

\section{de intervenção psicológica}

Diversos estudos apontam que o abuso sexual vivenciado na infância e na adolescência, se não amparado adequadamente, aumentam o risco de futuros distúrbios psíquicos na vida adulta (Rush, 2008). O leque de possibilidades de atuação e intervenção vai desde o acontecimento, até o espaço que se dá entre a denúncia e as etapas de investigação e cuidado às vítimas. O apoio psicológico insere-se como importante ferramenta de cuidado e enfrentamento.

O abuso sexual precisa ser considerado de acordo com as implicações ocasionadas na saúde física e mental da criança e do adolescente, destacando sempre a importância do acolhimento e abordagens adequadas para o devido acompanhamento, de forma a minimizar e ressignificar essas sequelas.

Os efeitos psíquicos são preocupantes e os problemas decorrentes do abuso podem persistir na vida adulta dessas crianças, visto como fator de risco para a vitimização sexual na idade adulta (Abrapia, 2002) e para o desenvolvimento de psicopatologias futuras (Minayo, 2002). Relata-se que quanto mais frequentes e persistentes forem os abusos, piores serão os problemas psíquicos, comportamentais e de relacionamento a serem desenvolvidos (Morales e Schramm, 2002). Dentre os principais problemas estão: medo, perda de interesse pelos estudos e brincadeiras, dificuldades de se

\footnotetext{
${ }^{6}$ Conselho Federal de Psicologia.
} 
ajustar, isolamento social, déficit de linguagem e aprendizagem, baixa autoestima (Gabel, 1997), uso de álcool e drogas, ideias suicidas e homicidas, tentativas repetidas de suicídio, automutilação e agressividade. Porém, ressalta-se que algumas crianças e adolescentes, apesar do sofrimento vivenciado, passam por essa experiência sem apresentar o quadro de impactos descritos pela literatura, de forma que conseguem encontrar "caminhos de (re)construção de suas próprias vidas" (Deslandes, 2003).

A criança deve ser acolhida e ser conscientizada de que ela é sujeito e não objeto da ação sofrida, e que embora exista o processo de vitimização sofrido, alguns recursos internos podem ser explorados para enfrentamento (Viaux, 1997). A Psicologia com seus aparatos de ciência e profissão tem o importante papel para contribuir substancialmente na eficácia dessas medidas de prevenção.

O Conselho Federal de Psicologia elaborou o documento: Serviço de Proteção Social a Crianças e Adolescentes Vítimas de Violência, Abuso e Exploração Sexual e suas Famílias: referências para a atuação do psicólogo, com diretrizes básicas para a atuação dos profissionais de psicologia frente a esses casos. De acordo com o referido relatório, o atendimento psicológico deve compor a atenção psicossocial, tendo por princípio a prioridade absoluta, em razão da condição peculiar de desenvolvimento dos sujeitos. Portanto, em casos de abuso sexual contra crianças e adolescentes o trabalho precisa estar voltado para o fortalecimento e o restabelecimento de direitos das crianças e/ou adolescentes à convivência familiar e comunitária, em condições dignas de vida (CFP, 2009).

Ações interventivas precisam estar pautadas na possibilidade de superação da situação de violação de direitos, além da reparação da violência sofrida, integrando a rede de cuidados, considerando sempre a fase e desenvolvimento na qual as vítimas se encontram, possibilitando assim, a interrupção do ciclo da violência para a redução de danos sofridos e para a construção de condições de proteção e de autonomia.

A revisão de literatura feita por Padilha e Gomide (2004), permitiu o apontamento de sete principais motivos de se fazer uma prevenção terciária em crianças e adolescentes vítimas de abuso sexual, dentre elas destaque-se: 1) elas tendem a tornarem-se reprodutoras de 
maus tratos na vida-adulta; 2) apoio diante de uma situação reveladora diminui as sequelas emocionais; 3) antes de uma revelação a público, deve-se passar por um acolhimento empático e não crítico; 4) a grupoterapia facilita o rompimento do segredo e o isolamento; 5) prevenções de esquiva pela diminuição da ansiedade; 6) a livre expressão facilita a percepção e modificação de culpa que o sujeito tem de si e 7) prevenção da revitimização. Tais pressupostos só vêm reafirmar e embasar a importância da prática do amparo psicológico terciário às emoções e comportamentos das crianças e adolescentes abusadas sexualmente, proporcionando uma visão ampla do acontecimento e melhorando assim o repertório de enfrentamento dos pacientes.

A terapia cognitivocomportamental tem sido utilizada nas mais diversas práticas e intervenções em saúde, tanto no enfoque individual, como também em grupo. Há décadas, tem sido afirmada a eficácia de suas intervenções em diversos âmbitos, com os estudos dos percussores Aaron Beck e Albert Ellis (Knapp, 2004). Tem-se mostrado uma abordagem com alto grau de adaptabilidade a pacientes com diferentes níveis de idade, renda e educação (Beck, 2013). Mostra-se embasada com intervenções breves e objetivas, recomendada nos últimos anos, como uma abordagem propicia ao tratamento de inúmeros casos na rede pública (Silva, Pereira e Aquino, 2011).

Inicialmente, a história da TCC traz como foco central o tratamento de casos de ansiedade e depressão (Clark e Beck, 2012), porém sua aplicabilidade tem sido estendida atualmente a diversas áreas, desde cuidados primários e outras especializações da saúde, as escolas, prisões, dentre outros ambientes e contextos (Beck, 2013).

\section{A Terapia Cognitivo-} comportamental de acordo com Beck (2013) baseia seu tratamento na compreensão ou conceituação de cada sujeito, especificamente, suas crenças e padrões de comportamentos. O terapeuta, objetiva produzir uma mudança cognitiva (pensamentos e crenças do paciente), para que haja uma mudança emocional e comportamental permanente. Assim, pensamentos disfuncionais que possam ser prejudiciais aos indivíduos como: constantes fracassos, desmotivação, baixa autoestima e depressão em decorrência do sofrimento, tornam-se 
ciclos viciosos em que a TCC busca interromper, ensinando aos pacientes maneiras efetivas de equilibrar tais prejuízos do transtorno e manejar as evitações (Safren et. al., 2008; Barkley, 2010,).

Assim, nessa concepção cognitivista, o modelo da Terapia Cognitiva Comportamental é bem conceituado por Petersen e Wainer como:

[...] uma abordagem terapêutica bem estruturada, diretiva com metas claras e definidas, focalizada no presente e utilizada no tratamento dos mais diferentes transtornos psicológicos. Seu objetivo principal é o de produzir mudanças nos pensamentos e nos sistemas de significados (crenças) dos clientes, evocando uma transformação emocional e comportamental duradouras, e não apenas um decréscimo momentâneo dos sintomas (2011, p.18).

No contexto do abuso sexual, a criança e o adolescente que passa pelo processo de psicoterápico cognitivistacomportamental deve aprender mecanismos para se recolocar em seu curso normal de vida, através de um repertório de enfrentamento com habilidades emocionais e sociais importantes para melhorar sequelas de abuso e seus efeitos (Padilha e Gomide, 2004). Em um estudo feito por
Hanbigzang (2009), as pesquisas que utilizam a terapia cognitivocomportamental como enfoque, têm obtido melhores resultados quando comparadas com outras terapias não diretivas para crianças e adolescentes com problemas comportamentais advindos do abuso sexual.

\section{Técnicas cognitivas e comportamentais em grupoterapias de apoio a crianças e adolescentes vítimas de abuso sexual}

A terapia grupal de crianças e adolescentes vitimadas pelo abuso sexual, permite que os pacientes através de suas experiências num contexto de vivências coletivas, enxerguem suas dificuldades e tudo que está ligado direta ou indiretamente a ela, desde pensamentos disfuncionais, que possam gerar emoções negativas, até comportamentos prejudiciais que estejam paralisando a vida desses sujeitos. Mencionarei neste capítulo o estudo feito por Hanbigzang e colaboradores em diversos anos, onde a grupoterapia objetivou à redução de sintomas de depressão, ansiedade e transtorno do estresse pós-traumático, 
bem como à reestruturação de crenças disfuncionais em relação à experiência abusiva.

De acordo com Neufeld (2011), existem quatro modalidades de intervenções cognitivo-comportamentais em grupo: 1) psicoeducação, 2) grupos de apoio, 3) grupos de orientação e/ou treinamento e 4) grupos terapêuticos. No contexto da TCCG - seja qual for a modalidade escolhida ou a temática a ser trabalhada - o tratamento de um número maior de pacientes em um único atendimento, permite a agilidade na demanda das instituições, como também a diminuição dos altos custos concernidos na rede pública (Neulfeld, 2011). Traremos a seguir algumas técnicas utilizadas em atendimentos grupoterápicos em terapias cognitivocomportamentais, encontradas na literatura direcionada ao público estudado. A apresentação se estrutura de modo a contemplar a técnica utilizada, suas características e os possíveis resultados encontrados.

- Psicoeducação;

- Treinamento de Inoculação de Estresse (TIE);

- Prevenção à recaída;
Etapa 1- Psicoeducação; A Terapia Cognitivo-Comportamental pode ensinar habilidades para modificar pensamentos e gerenciar estados de humor, assim como mudar comportamentos de forma significativa (Aguiar e Dantas, 2018). Chamamos essa forma de ensinamento de psicoeducação e é usada em diversos contextos, desde a psicologia, pedagogia ou a medicina.

A psicoeducação trata-se de uma educação do paciente em relação a sua demanda/diagnóstico, corroborando assim, um dos princípios fundamentais da TCC, de que o paciente é capaz de aprender habilidades e modificar pensamentos disfuncionais, tornando-se capaz de alinhar e modificar comportamentos. Tais habilidades são encontradas e aperfeiçoadas na psicoeducação, momento este da psicoterapia, imprescindível que instrumentaliza o paciente a conhecer e praticar todo conhecimento adquirido sobre sua problemática. Reduzindo assim, o risco de uma recaída (Wright, Basco e Thase, 2008).

Em grupos, a psicoeducação possibilita a criação de espaços nos quais as pessoas podem reconhecer as suas dificuldades, pensamentos, emoções, e 
comportamentos relacionados a elas. (Oliveira, et. al, 2018). É possível que um paciente tenha mais autonomia no processo terapêutico na medida em que se sente capaz de identificar e mudar pensamentos, manejar emoções e quebrar padrões de comportamentos que não o ajudam. (Aguiar e Dantas, 2018).

\section{Etapa 2- Treinamento de Inoculação}

de Estresse (TIE); O TIE é realizado na perspectiva de controle das emoções pertinentes às lembranças do trauma $\mathrm{e}$ reestruturação da memória traumática. (Schneider e Habigzang, 2016).

No estudo de Hanbigzang et. al. (2009), o treino de inoculação de estresse é composto por quatro sessões, onde ele traz técnicas em que o paciente apresente e treine de forma gradual, as situações abusivas experienciadas pelo relato oral ou por escrito; faça uma reestruturação cognitiva das memórias traumáticas; relate detalhadamente o abuso sexual; mapeie a frequência e a intensidade das lembranças do abuso sexual e dos eventos desencadeadores destas lembranças; treine a substituição de imagens positivas e negativas; relate o pior momento do abuso sexual; construa estratégias cognitivas e comportamentais, para num momento de emergência saber lidar com lembranças intrusivas do abuso.

Ainda de acordo com Hanbigzang et. al. (2009), na aplicação do TIE é imprescindível o apoio total do psicoterapeuta grupal, diante das repercussões negativas, pensamentos, emoções e comportamentos que serão (re)cordados e (re)sentidos, trazendo as vítimas, momentos difíceis, mas que posteriormente, trarão a reestruturação da memória traumática.

\section{Etapa 3- Prevenção à recaída; A} prevenção à recaída é uma etapa indispensável para se fechar com sucesso um acompanhamento grupal de crianças e adolescente vítimas de abuso sexual. Através dela, o paciente aprende habilidades de autoproteção para prevenir futuras revitimizações (Hanbigzang, 2009). Nesta fase da terapia, Maia (2001), também vem ressaltar a importância do trabalho com a imagem corporal do cliente, bem como uma orientação voltada para o futuro, incentivando a tomada de decisão perante suas escolhas, seu bem-estar físico e a relação com as outras pessoas.

Num estudo feito por Hanbigzang et al. (2009), ele descreve seis sessões durante a etapa de prevenção 
a recaída da seguinte forma: Oficina educação sexual, na qual são abordadas questões referentes ao autocuidado, mudanças físicas da puberdade $\mathrm{e}$ métodos contraceptivos; Oficina de psicomotricidade; Oficina sobre o Estatuto da criança e do adolescente, Apresentação e discussão do vídeo Estatuto do Futuro Dramatização de audiência (abordar a possibilidade de participação de audiências, esclarecendo dúvidas e preparando as participantes para tal situação); Treino de habilidades sociais focadas em medidas protetivas (identificação de situações de risco ensaio cognitivo e comportamental de estratégias de proteção contra futuras revitimizações), Escolha de um adultoreferência para solicitar ajuda em situações de risco; Retomada das estratégias cognitivas e comportamentais aprendidas no contexto grupal, Abordagem das perspectivas com relação ao futuro das participantes e reestruturar possíveis crenças distorcidas; Auto-avaliação por meio de registro escrito das mudanças percebidas antes e depois da grupoterapia em relação a si, como se relaciona com os outros e visão do futuro.

Ao término desta etapa, a criança ou o adolescente se torna capaz de idealizar vários projetos de vida, a nível pessoal e interpessoal, ter autonomia de pedir ajuda se necessário, se cuidar e deixar ser cuidada (Maia, 2001).

\section{Considerações Finais}

A TCC é utilizada como forma de tratamento por ter um modelo de ações que se mostram bastantes eficazes ao longo do processo psicoterápico. Verificou-se que a TCC cumpre seu objetivo diante da demanda de crianças e adolescentes com transtornos ansiosos pós vivenciar traumas complexos, reduzindo sintomas e sendo corresponsável pela diminuição de recidivas.

A terapia cognitivocomportamental mediante uso das técnicas de psicoeducação, treinamento de inoculação de estresse e prevenção à recaída, mostrou ótimos resultados nos pacientes que no início do tratamento apresentaram alto índice de ansiedade, medo e culpa. Assim, com a aplicação das técnicas e apoio do psicoterapeuta é possível que os pacientes tenham um maior controle de seus pensamentos, emoções e saibam reconhecer momentos em que possam ter autonomia e 
conhecimento de reconhecer como, quando e a quem pedirem ajuda.

$\mathrm{Na}$ literatura explorada verificouse que ainda há uma escassez no que se refere a estudos da terapia cognitivocomportamental em grupo - (TCCG) e suas técnicas com ênfase em grupos infanto-juvenis que abordam o tema abuso sexual. Sendo assim, propomos que outros estudos sejam feitos, a partir de estudos de casos reais, para que pesquisadores, especialistas e estudiosos em geral, possam avaliar na prática a eficácia da TCC mediante o abuso sexual.

\section{Referências}

Abrapia (2002), “Associação Brasileira Multiprofissional de Proteção à infância e à adolescência", Consultado em: http://www.abrapia.org.br.

Aguiar, Camita Stor de; Dantas, Nathália Della Santa Melo (2018), Baralho do modelo cognitivo para adultos: psicoeducação dos pensamentos, sentimentos e comportamentos. Rio de Janeiro: Sinopsys Editora e Sistemas Ltda.

Araújo, Maria de Fátima (2002), "Violência e abuso sexual na família”, Psicologia em Estudo, 7(2), 311.

Barkley, Russel (2010), Transtorno de déficit de atenção e hiperatividade em adultos: a mais recente avaliação e estratégias de tratamento. Massachusetts: Jones and Bartlett Publishers.

Barros, W. S (2004), "Crimes contra os costumes - Estupro", In Programa Delegacia Legal, Secretaria de Segurança Pública, Governo do Estado do Rio de Janeiro. Rio de Janeiro; SSP/RJ, 14-19.

Beck, Judith (2013), Terapia cognitivocomportamental: teoria e prática, 2ed. Porto Alegre: Artmed.

Brasil. Fundação ABRINQ (2017), A Criança e o adolescente nos objetivos de desenvolvimento sustentável (ODS) Marco zero dos principais indicadores brasileiros ODS 1, 2, 3 e 5. São Paulo. Consultado em: https://fadc.org.br/sites/default/files/201 9-02/cenario-brasil-2018.pdf.

Brasil, Ministério Público Do Distrito Federal e Territórios (2015), Violência sexual contra crianças e adolescentes: 
identificação e enfrentamento. Brasília: $1^{\mathrm{a}}$ Edição.

Brasil (1999), Plano Nacional de Enfrentamento a Violência sexual infanto-juvenil. 3ed. Ministério da Justiça. Brasília: SEDH/ DCA.

Brasil (2003), Estatuto da Criança e do Adolescente de 1990. 4. ed. Brasília: Saraiva.

Braun, Suzana (2002), A violência sexual infantil na família do silêncio à revelação do segredo. Porto Alegre: AGE editora.

Capitão, Cláudio Garcia; Romaro, Rita Aparecida (2008), Caracterização do abuso sexual em crianças e adolescentes, Psicol. Am. Lat., México, 13, jul.

Clark, David; Beck, Aron (2012), Terapia cognitiva para os transtornos de ansiedade. Porto Alegre: Artmed.

Conselho Federal de Psicologia CFP (2009), Serviço de Proteção Social a Crianças e Adolescentes Vítimas de Violência, Abuso e Exploração Sexual e suas Famílias: referências para a atuação do psicólogo. Brasília: CFP.

Deslandes, Suely (2003), “Atenção a crianças e adolescentes vítimas de violência doméstica: análise de um serviço", Cadernos de Saúde Pública, 10(01), 177-187.

Fundo das Nações Unidas para a Infância UNICEF (2005), Pequenas Vítimas. Relatório UNICEF - Situação da Infância Brasileira 2006. Brasília: UNICEF. Consultado em: http://www.unicef.org/brazil/pt/.

Gabel, Marceline (Org.) (1997), Crianças vítimas de abuso sexual. São Paulo: Summus.

Gerhardt, Tatiana Engel; Silveira, Denise Tolfo (2009), Métodos de pesquisa. Porto Alegre, Editora da UFRGS.

Gil, Carlos Alberto (2010), Métodos e técnicas de pesquisa social. São Paulo: Editora Atlas.

Habigzang, Luísa; AZEVEDO, Gabriela Azen; Koller, Silvia Helena; Machado, Paula Xavier (2006), "Risk and protective factors in the resource network for children and adolescences victims of sexual violence", Psicologia: Reflexão e Crítica, 19(03), 379-386.

Habigzang, Luísa; Koller, Silvia Helena (2011), "Terapia cognitivocomportamental para crianças e 
adolescentes vítimas de abuso sexual", In: Petersen, C. S. Wainer, R. (Cols.) (2011). Terapias cognitivocomportamentais para crianças e adolescentes. Porto Alegre: Artmed.

Hanbigzang, Luísa et al (2009), "Grupoterapia cognitivocomportamental para crianças e adolescentes vítimas de abuso sexual", Rev. Saúde Pública, 43(01), 70-8.

Knapp, Paulo (2004), Terapia cognitivocomportamental na prática psiquiátrica. Porto Alegre: Artmed.

Lira, Margaret Olinda de Souza Carvalho et al (2017), “Abuso sexual na infância e suas repercussões na vida adulta”, Texto-contexto enferm. Florianópolis, 26(03).

Maia, Angela (2001), Abuso sexual na infância: a reconstrução depois do trauma. Instituto de educação e psicologia, Universidade de Minho, Portugal: Psicologia: teoria investigação e prática.

Marconi, Maria de Andrade; Lakatos, Eva Maria (2010), Fundamentos de Metodologia Científica. 7ed. São Paulo: Editora Atlas.
Minayo, Maria Cecília de Souza (2002), “O significado social e para a saúde da violência contra crianças e adolescentes", In: Westphal, M. F. Violência e criança. São Paulo: EDUSP.

Brasil, Ministério da Justiça (2018), Denúncias feitas ao Disque 100 Módulo crianças e adolescentes segundo tipos de violação e sexo. Consultado

em: https://observatoriocrianca.org.br/cenari o-infancia/temas/violencia/630denuncias-feitas-aodisque-100-modulocriancas-e-adolescentes-segundo-tiposde-violacao-esexo?filters $=1,258$

Morales, Álvaro; Schramm, Fermin (2002), “A moralidade do abuso sexual intrafamiliar em menores", Ciência \& Saúde coletiva, 7(02), 265-273.

Moreschi, Marcia Teresinha (2018), Violência contra Crianças e Adolescentes: Análise de Cenários e Propostas de Políticas Públicas. Brasília: Ministério dos Direitos Humanos.

Neufeld, Carmem Beatriz (2011), "Intervenções em grupos na abordagem cognitivo-comportamental”, In: Rangé, Bernard, Psicoterapias cognitivo- 
comportamentais: Um diálogo com a psiquiatria. 2ed. Porto Alegre: Artmed.

Oliveira, Polliana Rodrigues de; Menezes, Marcela Barbosa de; Brito, Soraia Silva; Pinto, Paula Sanders Pereira (2018), "Psicoeducação das emoções e habilidades sociais: uma proposta de promoção e prevenção de saúde mental para adolescentes", XVII SEPA - Seminário Estudantil de Produção Acadêmica, UNIFACS.

Organização Mundial de Saúde OMS (2002), Relatório Mundial sobre Violência e Saúde. Genebra: Organização Mundial de Saúde.

Padilha, Maria da Graça Saldanha; Gomide, Paula Inês Cunha (2004), "Descrição de um processo terapêutico em grupo para adolescentes vítimas de abuso sexual", Estudos de psicologia, 9(01), 53-61.

Pelisoli, Cátula; Piccoloto, Luciane Benvegnu (2010), "Prevenção do abuso sexual infantil: Estratégias cognitivocomportamentais na escola, na família e na comunidade", Revista Brasileira de Terapias Cognitivas, 6(01).

Petersen, Circe Salcides; Wainer, Ricardo (Cols.) (2011), Terapias cognitivo-comportamentais

para crianças e adolescentes. Porto Alegre: Artmed.

Pinheiro, Paulo Sergio (2016), Relatório do especialista independente sobre o Estudo das Nações Unidas sobre Violência contra Crianças, Distribuição Geral. Consultado em: http://www.unicef.org/brazil/Estudo_PS P_Portugues.pdf.

Ribeiro, Maria Aparecida; Ferriani, Maria das Graças Carvalho; Reis, Jair Neves dos (2004), "Violência sexual contra crianças e adolescentes: características relativas à vitimização nas relações familiares", Cadernos de Saúde Pública, 20(02), 456-464.

Rush, Florence (2008), The best kept secret: sexual abuse of children. New York: McGraw-Hill Book Company.

Saffioti, Heleieth (1997), "No fio da navalha: violência contra crianças e adolescentes no Brasil atual”. In: Madeira, Felícia Reicher (Org.). Quem mandou nascer mulher?. São Paulo: Editora Rosa dos Tempos.

Safren, Steven; Perlman, Carol; Sprith, Susan; Otto, Michael (2008), Dominando o TDA/H adulto: programa 
de tratamento cognitivo-comportamental (guia do terapeuta). Porto alegre: Artmed.

Santos, João Diógenes Ferreira dos. (2010), "Rompendo O Silêncio": violência sexual contra meninas no espaço doméstico. In: $9^{\circ}$ Fazendo Gênero: Diásporas, Diversidades, Deslocamentos.

Schneider, Jaluza Aimèe; Habigzang, Luísa Fernanda (2016), “Aplicação do programa cognitivo comportamental superar para atendimento individual de meninas vítimas de violência sexual: estudos de caso", Avances en Psicología Latinoamericana, 34(03), 543-556.

Seabra, André Salame; Nascimento, Helena Maria do (1998), “Abuso sexual na infância", Arquivos Brasileiros de Pediatria, 34(07), 395-415.

Silva, Shirley de Souza; Pereira, Reginete Cavalcanti; Aquino, Thiago Antônio Avellar (2011), “A terapia cognitivo-comportamental no ambulatório público: possibilidades e desafios", Revista Brasileira de Terapias Cognitivas, 7(01), 44-49.

Viaux, Jean-Luc (1997), “A perícia psicológica das crianças vítimas de abusos sexuais", In: Gabel, Marceline (Org.), Crianças vítimas de abuso sexual. São Paulo: Summus.

Viodres Inoue, Silvia Regina; Ristum, Marilena (2008), "Violência sexual: caracterização e análise de casos revelados na escola", Estudos de Psicologia. Campinas, 25(01), 1-21.

Wright, Jesse; Basco, Monica Ramires; Thase, Michael (2008), Aprendendo a terapia cognitivo-comportamental: um guia ilustrado. Porto Alegre: Artmed.

Zagaglia, Rosangela (2004), "Crimes contra crianças e adolescentes", In: Pisá, Graça; Barbosa, Gabriela Ferrarese (Orgs.). A violência silenciosa do incesto. Rio de Janeiro: Imprensa Oficial 